\title{
A first study of the galaxy HRG 2304 and its companion AM 1646-795 (NED01) ${ }^{\star}$
}

\author{
E. Wenderoth ${ }^{1}$, M. Faúndez-Abans ${ }^{2}$, A. C. Krabbe ${ }^{3}$, M. de Oliveira-Abans ${ }^{2}$, and H. Cuevas ${ }^{4}$ \\ ${ }^{1}$ Gemini Observatory, Southern Operation Center, c/o AURA, Casilla 603, La Serena, Chile \\ e-mail: ewenderothagemini .edu \\ 2 Laboratório Nacional de Astrofísica, Rua Estados Unidos 154, 37504-364, Itajubá, MG, Brazil \\ e-mail: max@lna.br; mabans@lna.br \\ 3 Universidade do Vale do Paraíba - UNIVAP, Av. Shishima Hifumi 2911, Urbanova CEP: 12244-000, São José dos Campos, SP, \\ Brazil \\ e-mail: angela.krabbe@gmail.com \\ ${ }^{4}$ Universidad de La Serena, Benavente 980, La Serena, Chile \\ e-mail: hcuevas@dfuls.cl
}

Received 23 February 2011 / Accepted 21 March 2011

\begin{abstract}
Aims. We report the first study of the peculiar ring-like galaxy HRG 2304 (NED02), which was previously classified as a ring galaxy with an elliptical smooth ring. This object was selected to prove that it is a candidate for the Solitaire-type ring galaxies in an early stage of ring formation. The main goal of this work is to provide the spectral characteristics of the current object and its companion AM 1646-795 (NED01).

Methods. The study is based on spectroscopic observations in the optical band to highlight the characteristics of this interacting galaxy. To investigate the star formation history of HRG 2304 we used the stellar population synthesis code STARLIGHT. The direct $V$ and $B$ broad band images were used to enhance some fine structures.

Results. Along the entire long-slit signal, the spectra of HRG 2304 and its companion resemble that of an early-type galaxy. We estimated a heliocentric systemic redshift of $z=0.0415$, corresponding to heliocentric velocities of $12449 \mathrm{~km} \mathrm{~s}^{-1}$ for HRG 2304 (NED02) and $12430 \mathrm{~km} \mathrm{~s}^{-1}$ for AM1646-795 (NED01). The spatial variation in the contribution of the stellar population components for both objects are dominated by an old stellar population $2 \times 10^{9}<t \leq 13 \times 10^{9}$ yr. The observed radial-velocity distribution and the fine structures around HRG 2304 suggest an ongoing tidal interaction of both galaxies.

Conclusions. The spectroscopic results and the morphological peculiarities of HRG 2304 can be adequately interpreted as an ongoing interaction with the companion galaxy. Both galaxies are early-type, the companion is elliptical, and the smooth distribution of the material around HRG 2304 and its off-center nucleus in the direction of AM1646-795 (NED01) characterize HRG 2304 as a Solitairetype galaxy candidate in an early stage of ring formation.
\end{abstract}

Key words. galaxies: individual: HRG 2304 - methods: observational - techniques: spectroscopic - galaxies: interactions galaxies: general - galaxies: peculiar

\section{Introduction}

In the past decades, studies have shown that the gravitational interaction is the most important factor in galactic evolution. Gravitational interaction directly affects properties such as size, morphological type, luminosity, star formation rate (SFR), and mass distribution in galaxies. Gravity is the acting force that makes galaxies interact, collide, and merge. Colliding galaxies are twisted and deformed by their mutual gravitational fields. This often gives galaxies peculiar shapes and can create tails, plumes, and bridges between the interacting objects. These features are the patterns of the gas, dust, and some stars, which have been drawn out by tidal forces during interaction. In addition, interactions are seen in galaxies that form rings. An intruder galaxy can plunge through the center (or close to it) of a rotating disk galaxy, triggering the birth of bright young stars in the wake of radially expanding annular shock waves. Those objects are called ring galaxies (RGs) and belong to the morphological

\footnotetext{
* Based on observations performed with the telescope of $3.6 \mathrm{~m}$ at the European Southern Observatory (ESO), La Silla, Chile.
}

category 6 of the "Catalogue of Southern Peculiar Galaxies and Associations" (see Arp \& Madore 1977, 1986, as a starting point in the study of peculiar galaxies with rings). In general, the rings may either be collisional (peculiar - pRG) or resonant (normal NRG, see Faúndez-Abans \& de Oliveira-Abans 1998a - hereafter FAOA, for this denomination). It is well accepted that collisional RGs are important regions for studying disks that remain fairly intact after the interaction, allowing detailed observation of their structures and star formation properties, such as galaxyscale perturbation properties, hydrodynamics, vigorous nonnuclear star formation, and stellar evolutionary processes across and inside the ring. A description of morphological characteristics of pRG is found in FAOA. General properties of those galaxies can be found in Theys \& Spiegel $(1976,1977)$, Lynds \& Toomre (1976), Appleton \& Struck-Marcell (1996), and for a short review see Dennefeld \& Materne (1980).

In the work on the morphology of pRGs by FAOA, the morphological categories are compressed into five families, following the general behavior of the galaxy-ring structures. Eight morphological subdivisions are highlighted in Table 1 of that 
Table 1. Stars very near to HRG 2304 and two field galaxies.

\begin{tabular}{llll}
\hline \hline Object & RA $(2000)$ & Dec $(2000)$ & Remarks \\
\hline Star & $16^{\mathrm{h}} 54^{\mathrm{m}} 40^{\varsigma} .47$ & $-80^{\circ} 03^{\prime} 43^{\prime \prime} 98$ & GSC094100288 \\
Star & 165439.92 & -800351.77 & The projected anonymous star on HRG 2304 \\
Galaxy & 165423.47 & -800353.38 & 2MASX J16542367-8003537 \\
Star & 165437.56 & -800423.99 & Anonymous star south of C (NED01) \\
Galaxy & 165530.55 & -800547.92 & 2MASX J16553050-8005482 $(a)$ \\
\hline
\end{tabular}

Notes. ${ }^{(a)}$ Galaxy with $12465 \mathrm{~km} \mathrm{~s}^{-1}, z=0.0415$, see NED.

paper. One of these morphological subdivisions is a basic structure called "Solitaire". The pRG Solitaire is described as an object with the bulge on the ring, or very close to it, resembling a one-diamond finger ring (single knotted ring). In these objects, the ring generally looks smooth and almost thin on the opposite side of the bulge (as archetypes see FM 188-15/NED02 and AM 0436-472/NED01). In spite of the poor statistics, the companion galaxy may be almost elliptical. In Faúndez-Abans et al. (2011, in prep.), a preliminary study and statistics of solitaire-like objects are carried out with the existing cataloged pRG-solitaire type objects.

No interacting galaxies in the early stage of formation of a Solitaire-type morphology are reported in the literature, so the galaxy HRG 2304 was selected as a candidate. In this paper, we report the first results of a study of the tidally disturbed galaxy HRG 2304 (NED02) and its companion AM 1646-795 (NED01) based on data obtained from long-slit spectrophotometric observations, at ESO-La Silla Observatory, in Chile.

\section{Early data and the field around HRG 2304}

The galaxy HRG 2304 looks like an elliptical galaxy on the Digitized Sky Survey (DSS) (see also: the film copies of the ESO/UPPSALA survey of the ESO(B) atlas, FAOA and NED for more references). As previously mentioned, no dedicated study has been done on this object and it appears only in a few references: (a) in a list of probable ring galaxies in a limiting magnitude of 17.5 in the $J$ band (Faúndez-Abans et al. 1994); (b) in a list of morphological characteristics of pRG by FAOA; (c) in the 2MASS-selected flat galaxies catalog (Mitronova et al. 2004); and (d) in the catalog of near-infrared properties of LEDA galaxies by Paturel et al. (2005).

The field of HRG 2304 in Fig. 1 shows in the center three objects aligned from the N-S direction. The first one is a star (GSC094100228 of $14.26 \mathrm{~V} \mathrm{mag}$ ), the second is HRG 2304 (NED02) and its extended halo-ring-like structure, and the last one is the galaxy AM 1646-795 (NED01). After enhancing the original image by filtering processing (Faúndez-Abans \& de Oliveira-Abans 1998b), Fig. 2 shows a zoom of the field around HRG 2304 in the $V$ band to look for structures in the bulge of HRG 2304. A projected star on HRG 2304 is clearly separated from the galaxy bulge on the NW, and in the South of the $\mathrm{C}$ galaxy, a tinny blob is enhanced. Bad column pixels were enhanced by the procedure. Table 1 lists the stars very near HRG 2304 and two prominent field galaxies. In Fig. 3 some structures are highlighted in NED01 and NED02; see Sect. 5 for the description and discussion of both figures.

\section{Observations and data reduction}

The observations were made in the service mode at La Silla Observatory with the ESO 3.6-m telescope equipped with ESO

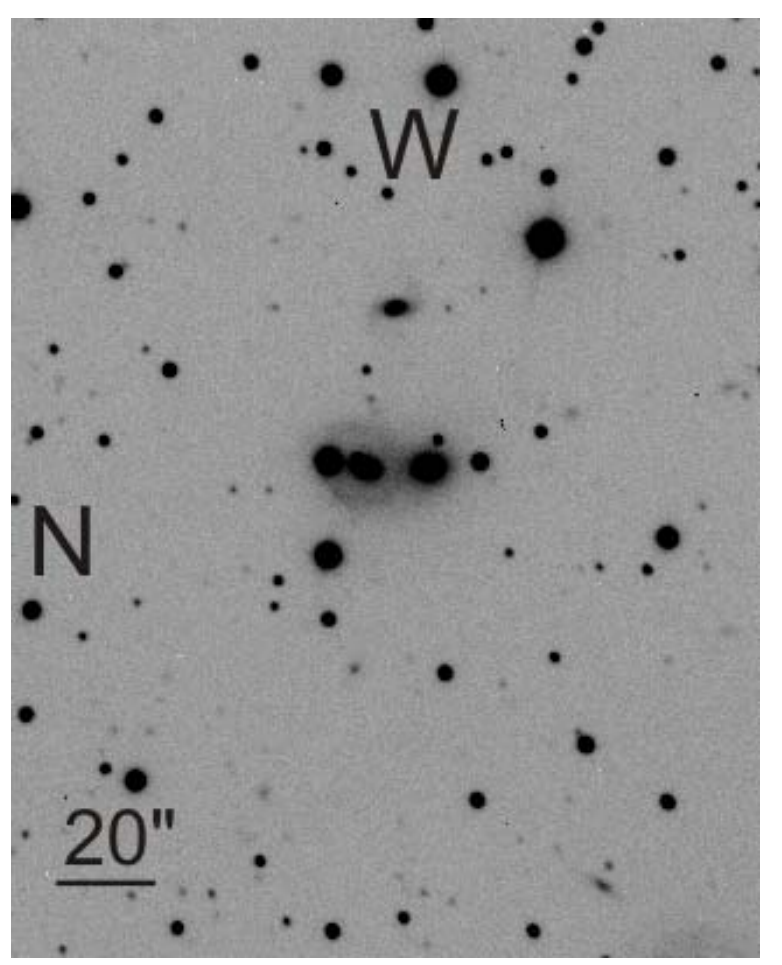

Fig. 1. Field around HRG 2304 in the $V$ band. West is up and north is to the left. There are two prominent objects aligned from $\mathrm{N}$ to $\mathrm{S}$, encompassing HRG 2304, from left to right: the star GSC094100228 of 14.26 V mag, HRG 2304, and the AM 1646-795 (NED01) galaxy.

Faint Object Spectrograph and Camera (EFOSC2). We used a CCD \# 40 LORAL/LESSER of $2048 \times 2048$ pixel, thinned and AR coated. A binning of $2 \times 2$ was adopted, implying a field on the plane of the sky of $2.68 \times 2 ! 68$. The grism Gr \#4 with a grating of 360 grooves $\mathrm{mm}^{-1}$ was used. The spectra obtained covered the wavelength range of 4085-7520 $\AA$ with a scale dispersion of $1.68 \AA$ pix $^{-1}$ and a $F W H M$ resolution of $14 \AA / 1^{\prime \prime}$. The slit width was set to $2^{\prime \prime}$ for the galaxies and to $5^{\prime \prime}$ for the standard stars. Two spectra of 720 and 600 s were obtained. The stars used for extinction and flux calibration are tertiary standards from Baldwin \& Stone (1984), as revised by Hamuy et al. (1992, see also Hamuy et al. 1994). The data reduction (bias correction, flat-fielding, cosmic ray cleaning, wavelength and flux calibrations, and 1D spectra extraction) was made using IRAF software.

\section{Analysis and results}

We report the first optical results for the galaxies HRG 2304 (NED02) and AM 1646-795 (NED01) using long-slit spectroscopy. The new results of velocity and $z$, as well as some early 


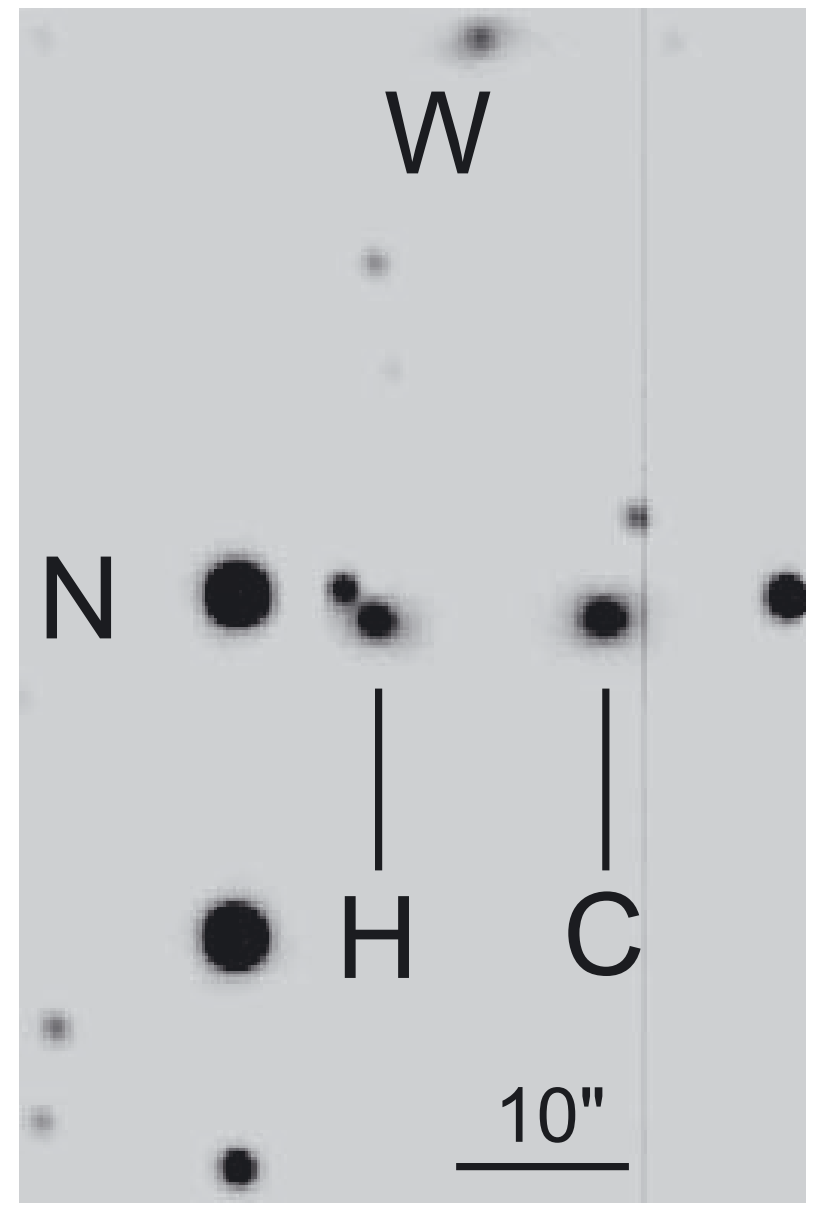

Fig. 2. A zoom of the field around HRG 2304 in the $V$ band. West is up and north is to the left. There is a star projected on the HRG 2304 galaxy near the bulge, $\mathrm{N}-\mathrm{W}$ direction. The image was enhanced using a median filtering of 30 square-pixel kernel (Faúndez-Abans \& de Oliveira-Abans 1998b). The H letter indicates HRG 2304 (NED02) and $\mathrm{C}$ is AM 1646-795 (NED01), the companion.

information on both galaxies are listed in Table 2. A value of $H_{\mathrm{o}}=70 \mathrm{~km} \mathrm{~s}^{-1} \mathrm{Mpc}^{-1}$ was adopted throughout this work.

\subsection{The spectra and the kinematics}

The spectra of both galaxies are shown in Fig. 4, in the range $4085 \AA$ to $7520 \AA$ A. It displays the main stellar features identified in the nuclear section of both galaxies: the absorption $\mathrm{G}$ band $\lambda 4305 \AA, \mathrm{H} \beta, \operatorname{MgIb} \lambda 5174 \AA, \mathrm{MgH} \lambda 5269 \AA$, NaID $\lambda 5892 \AA$, $\mathrm{H} \alpha$ and the $\mathrm{TiO}$ band in $\lambda \lambda 6250,7060 \AA$. Both spectra show features which are characteristic of late-type stars. The absorption and positions of these lines were determined by fitting a Gaussian to the observed profile.

For the calculation of the velocity, we considered the values corresponding to the maxima of the continuum intensity as systemic velocities. Using the MgIb and NaID absorption lines, the derived heliocentric systemic velocity $V_{\text {abs }}$ for HRG 2304 is $12449 \pm 90 \mathrm{~km} \mathrm{~s}^{-1}(z=0.0415)$ and for AM 1646-795 (NED01) it is $12430 \pm 40 \mathrm{~km} \mathrm{~s}^{-1}(z=0.0415)$. Both galaxies form a bound system with an approximate $\Delta V=19 \mathrm{~km} \mathrm{~s}^{-1}$. Table 2 also displays a correlated velocity ( $V_{\text {corr }}$ ) for both galaxies using the $\mathrm{G}, \mathrm{H} \beta, \mathrm{MgIb}, \mathrm{MgH}$ and NaID lines. We have estimated the masses based on a Virial relation employing the effective radius of each galaxy. The calculated distances and dynamical masses
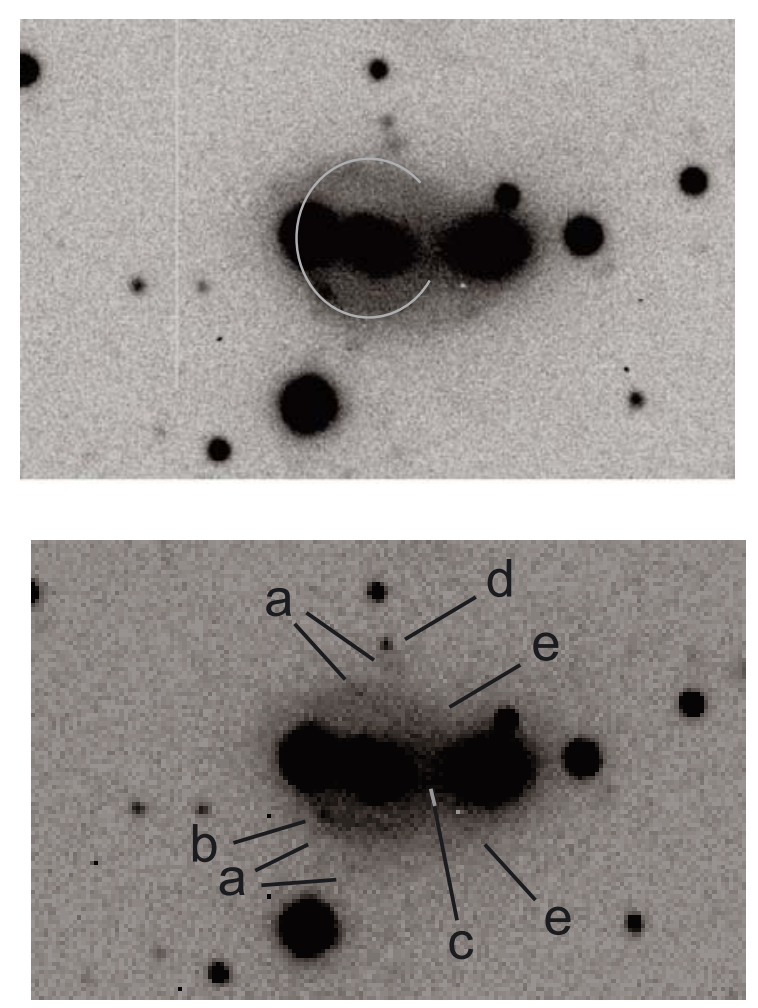

Fig. 3. Some structures are highlighted in NED01 and NED02. Top panel: the EFOSC2 direct $B$-filter image. Is drawn a ellipse-section of the ring. Lower panel: direct $V$-filter image (see Sect. 5 for the description of the a-e letters).

for NED01 and NED02 are 177.57 Mpc and 177.84 Mpc, $1.50 \times$ $10^{11} M_{\odot}$ and $0.71 \times 10^{11} M_{\odot}$, respectively. The adopted separation between both galaxy-centers is $13^{\prime \prime} .02$, and the radius of the ring-like structure of HRG 2304 is approximately 8.'7 long.

Figure 5 displays the rotation curve of both galaxies. The first panel shows the distribution for HRG 2304: open triangles and circles are data from MgIb lines (measurements from the two spectra); crosses are from the NaID lines, and filled stars stand for the correlated data from $\mathrm{G}, \mathrm{H} \beta, \mathrm{MgIb}, \mathrm{MgH}$ and NaID lines. The second panel shows the distribution for AM 1646-795 (NED01): crosses are data from the NaID lines, and open stars stand for the correlated data from $\mathrm{G}, \mathrm{H} \beta, \mathrm{MgIb}$, $\mathrm{MgH}$ and NaID lines. Figure 6 shows the rotation curve of both galaxies, from -2 '. 0 to 2 '. 0 around the kinematic center.

\subsection{Stellar population synthesis}

Detailed study of the star formation in tidally perturbed galaxies not only provides important information on the age distribution along their stellar population components, but also helps toward understanding several aspects related to the interacting process better, along with its effects on the properties of the individual galaxies and their evolution.

To investigate the star formation history of HRG 2304 and AM 1646-795 (NED01), we use the stellar population synthesis code STARLIGHT (Cid Fernandes et al. 2004, 2005; Mateus et al. 2006; Asari et al. 2007). This code is extensively discussed in Cid Fernandes et al. (2004, 2005), and is built upon computational techniques originally developed for empirical population synthesis with additional ingredients from evolutionary 
Table 2. Data on HRG 2304 (NED02) and AM 1646-795 (NED01) galaxies.

\begin{tabular}{|c|c|c|c|}
\hline Parameter & NED01 & NED02 & Ref. \\
\hline RA (2000) & 165439.20 & 165440.04 & (a) \\
\hline $\operatorname{Dec}(2000)$ & -800411.00 & -800347.35 & (a) \\
\hline pRG-family & & Solitaire-like & this work \\
\hline & 0.0415 & 0.0415 & this work \\
\hline$V_{\mathrm{abs}}\left(\mathrm{km} \mathrm{s}^{-1}\right)$ & $12430 \pm 40$ & $12449 \pm 40$ & this work \\
\hline$V_{\text {corr }}\left(\mathrm{km} \mathrm{s}^{-1}\right)$ & $12412 \pm 90$ & $12403 \pm 100$ & this work \\
\hline$z$ & 0.042 & & NED \\
\hline$V\left(\mathrm{~km} \mathrm{~s}^{-1}\right)$ & 12597 & & NED \\
\hline \multirow[t]{2}{*}{ Other designations } & 2MASX J16543910-8004109 & LEDA 228646 & NED \\
\hline & 6dFJ 1654393-800411 & 2MFGC 13532 & NED \\
\hline Magnitude & $16.5 \mathrm{~b}$ & $11.2 \mathrm{k}$ & NED \\
\hline Distance & $178 \mathrm{Mpc}$ & $178 \mathrm{Mpc}$ & this work \\
\hline Mass & $1.50 \times 10^{11} M_{\odot}$ & $0.71 \times 10^{11} M_{\odot}$ & this work \\
\hline Nucleus major axis $(B)$ & 2 .' 16 & 1.92 & this work \\
\hline Nucleus minor axis $(B)$ & 2.16 & $1 " .8$ & this work \\
\hline Bulge major axis $(B)$ & $9 ! 0$ & $7 ! \cdot 2$ & this work \\
\hline Bulge minor axis $(B)$ & 7".56 & 6!'72 & this work \\
\hline Ring major axis $(B)$ & & $15^{\prime \prime} 36$ & this work \\
\hline Ring minor axis $(B)$ & & $15^{\prime \prime} 36$ & this work \\
\hline$J-H$ & & 0.277 & NED \\
\hline$H-K$ & & 0.052 & NED \\
\hline$J-K$ & & 0.329 & NED \\
\hline
\end{tabular}

Notes. a: NED01 data from NED released after 20 abril 2009; NED02 data from Faúndez-Abans \& de Oliveira-Abans 1998b; $B$ : measurements in the $B$-filter image.
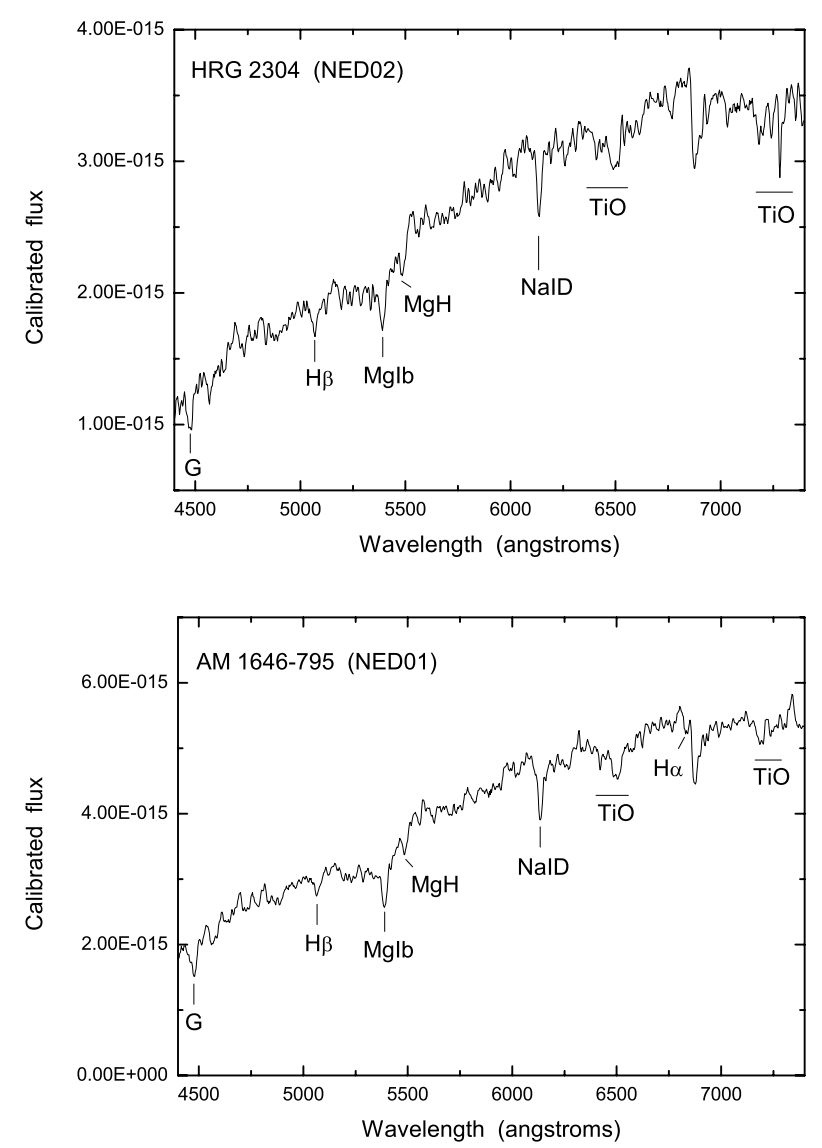

Fig. 4. Spectra of both galaxies. The vertical axis is the calibrated flux in units of $\mathrm{erg} \mathrm{s}^{-1} \mathrm{~cm}^{2} \AA^{-1}$. First panel: the nuclear features of HRG 2304. Second panel: same as the first panel, but for AM 1646-795 (NED01). Overplotted in both panels is the identification of the main absorption features: G band $\lambda 4305 \AA, \mathrm{H} \beta, \mathrm{MgIb} \lambda 5174 \AA, \mathrm{MgH} \lambda 5269 \AA$, NaID $\lambda 5892 \AA, \mathrm{H} \alpha$ and the TiO band in $\lambda \lambda 6250,7060$. synthesis models. This method was also used by Krabbe et al. (2011) and has been successful in describing the stellar population in interacting galaxies. Briefly, the code fits an observed spectrum with a combination of $N_{\star}$ single stellar populations (SSPs) from the Bruzual \& Charlot (2003) models. These models are based on a high-resolution library of observed stellar spectra, which allows for detailed spectral evolution of the SSPs across the wavelength range of 3200-9500 ^ with a wide range of metallicities. We used the Padova's 1994 tracks, as recommended by Bruzual \& Charlot (2003), with the initial mass function of Chabrier (2003) between 0.1 and $100 M_{\odot}$. Extinction is modeled by STARLIGHT as due to foreground dust, using the Large Magellanic Cloud average reddening law of Gordon et al. (2003) with $R_{V}=3.1$, and parametrized by the $V$-band extinction $A_{V}$. The SSPs used in this work cover 15 ages, $t=0.001$, 0.003, 0.005, 0.01, 0.025, 0.04, 0.1, 0.3, 0.6, 0.9, 1.4, 2.5, 5, 11, and $13 \mathrm{Gyr}$, and three metallicities, $Z=0.2 Z_{\odot}, 1 Z_{\odot}$, and $2.5 Z_{\odot}$, adding to 45 SSP components. The fitting is carried out using a simulated annealing plus Metropolis scheme, with regions around emission lines and bad pixels excluded from the analysis.

Figures 7 and 8 show an example of the observed spectrum corrected by reddening and the model stellar population spectrum for HRG 2304 and AM 1646-795 (NED01), respectively. The results of the synthesis are summarized in Table 3 for the individual spatial bins in each galaxy, stated as the perceptual contribution of each base element to the flux at $\lambda 5870 \AA$. Following the prescription of Cid Fernandes et al. (2005), we defined a condensed population vector, by binning the stellar populations according to the flux contributions into young, $x_{\mathrm{Y}}\left(t \leq 5 \times 10^{7} \mathrm{yr}\right)$; intermediate-age, $x_{\mathrm{I}}\left(5 \times 10^{7}<t \leq 2 \times 10^{9} \mathrm{yr}\right)$; and old, $x_{\mathrm{O}}$ $\left(2 \times 10^{9}<t \leq 13 \times 10^{9} \mathrm{yr}\right)$ components. The same bins were used to represent the mass components of the population vector $m_{\mathrm{Y}}, m_{\mathrm{I}}$, and $m_{\mathrm{O}}$ ). The metallicity $(Z)$, one important parameter to characterize the stellar population content, is weighted by light fraction. The quality of the fitting result is measured by the parameters $\chi^{2}$ and adev. The latter gives the perceptual mean 
E. Wenderoth et al.: The galaxy HRG 2304 and its companion
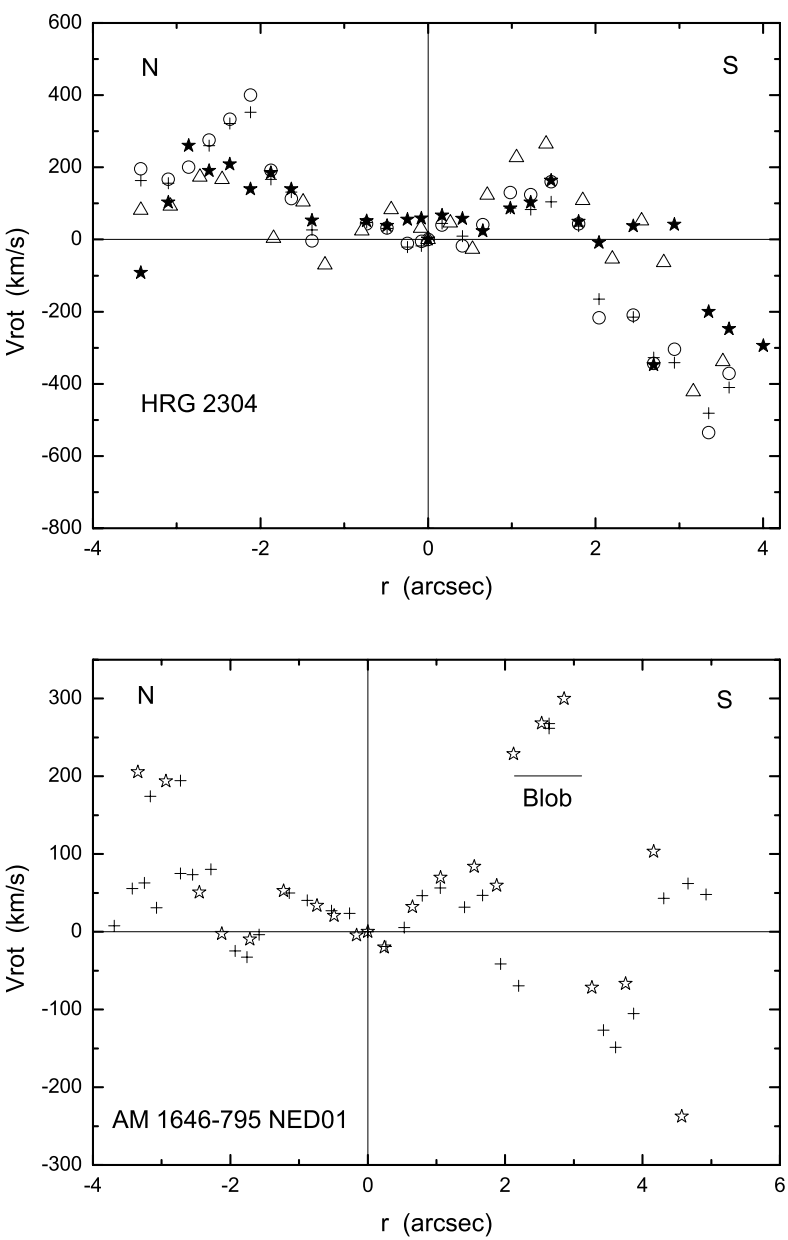

Fig. 5. The observed radial-velocity distribution for both galaxies. First panel is the distribution for HRG 2304: open triangles and open circles are data from MgIb lines (from the two spectra, respectively); crosses are from the NaID lines and filled stars stand for the correlated data from $\mathrm{G}, \mathrm{H} \beta, \mathrm{MgIb}, \mathrm{MgH}$ and NaID lines. Second panel is the same as the first one, but for AM 1646-795 (NED01): crosses are data from the NaID lines and open stars stand for the correlated data from $\mathrm{G}, \mathrm{H} \beta$, $\mathrm{MgIb}, \mathrm{MgH}$, and NaID lines.

deviation $\left|O_{\lambda}-M_{\lambda}\right| / O_{\lambda}$ over all fitted pixels, where $O_{\lambda}$ and $M_{\lambda}$ are the observed and model spectra, respectively.

The spatial variation in the contribution of the stellar population components are shown in Figs. 9 and 10 for the galaxies HRG 2304 and AM 1646-795 (NED01), respectively. As can be seen in Figs. 9 and 10, HRG 2304 and AM 1646-795 (NED01) are dominated by an old stellar population. A mean value of $Z=0.028$ was found for both galaxies.

\section{Discussion}

Along all the slit, the spectra of both galaxies show features which are characteristic of late-type stars. No star-forming regions and no nuclear ionization sources were detected. The two galaxies are a tidally bound system with a radial velocity difference of almost $19 \mathrm{~km} \mathrm{~s}^{-1}$. There are two nearby galaxies in the field: (a) 2MASX J16542367-8003537 without redshift data in the literature; and (b) 2MASX J16553050-8005482 with $z=0.0415,12465 \mathrm{~km} \mathrm{~s}^{-1}$. In this context, HRG 2304 and AM 1646-795 (NED01) are members of a nearby group of galaxies, and probably all the components are gravitationally bound. The HRG 2304 and AM 1646-795 (NED01) system is at
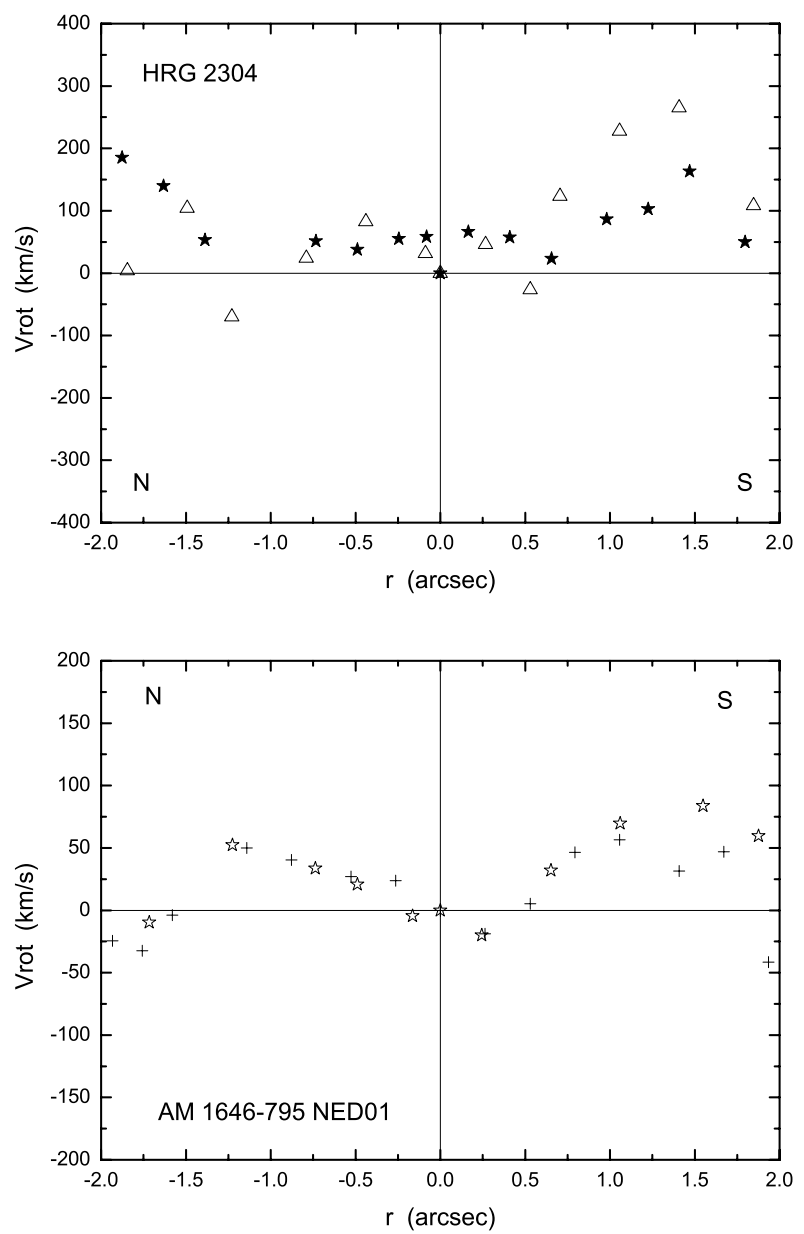

Fig. 6. The observed radial-velocity distribution for both galaxies from $-2{ }^{\prime \prime} 0$ to $22^{\prime \prime} 0$ around the kinematic center. First panel is the distribution for HRG 2304: open triangles are data from MgIb lines (from the two spectra, respectively); and filled stars stand for the correlated data from $\mathrm{G}, \mathrm{H} \beta, \mathrm{MgIb}, \mathrm{MgH}$ and NaID lines. Second panel is the same as the first, but for AM 1646-795 (NED01): crosses are data from the NaID lines and open stars stand for the correlated data from $\mathrm{G}, \mathrm{H} \beta$, $\mathrm{MgIb}, \mathrm{MgH}$, and NaID lines.

a distance of about $178 \mathrm{Mpc}$. From the quoted mass, HRG 2304 has approximately half of the mass of AM 1646-795 (NED01).

The errors of individual velocity measurements do not exceed $20 \mathrm{~km} \mathrm{~s}^{-1}$ in the central region of the galaxies and increase to $30-50 \mathrm{~km} \mathrm{~s}^{-1}$ on its periphery. There is a significant dispersion on the radial velocity distribution of HRG 2304 (see first panel of Fig. 5). The velocity dispersion are $\pm 40 \mathrm{~km} \mathrm{~s}^{-1}$ around the position of $\pm 11^{\prime \prime} 0$, and $\pm 90 \mathrm{~km} \mathrm{~s}^{-1}$ between a radius $\pm 22^{\prime \prime} 0$ to $\pm 44^{\prime \prime} 0$. With respect to the kinematic center, the north region is receding from us, while the one in the south, waved into receding (between a radius of $\sim 0$ !' $^{\prime} 1$ to 2. .' 0 ) and approaching us (between a radius of $\sim 22^{\prime \prime} 0$ to $4{ }^{\prime \prime} 0$ ). As in the first panel of Fig. 5, the radial velocity distribution of AM 1646-795 (NED01) varies in almost the same way as HRG 2304. There is an object tight-south to the very central region, which was detected by the luminosity profile in the spectral extraction (the Blob in the second panel of Fig. 5). The latter is receding from us and its velocity suggests that it is a satellite bound to NED01.

Inspecting Fig. 6, and following the variation of the radial velocity distribution it may be suggested that: (a) in the first panel, the nuclear region is approaching us; and (b) in the second panel, the nuclear region is receding from us. In both, the galaxy 
Table 3. Stellar-population synthesis results.

\begin{tabular}{|c|c|c|c|c|c|c|c|c|c|c|}
\hline $\begin{array}{l}\text { Pos. } \\
(\operatorname{arcsec})\end{array}$ & $\begin{array}{c}x_{\mathrm{Y}} \\
\text { (per cent) }\end{array}$ & $\begin{array}{c}x_{\mathrm{I}} \\
\text { (per cent) }\end{array}$ & $\begin{array}{c}x_{\mathrm{O}} \\
\text { (per cent) }\end{array}$ & $\begin{array}{c}m_{\mathrm{Y}} \\
\text { (per cent) }\end{array}$ & $\begin{array}{c}m_{\mathrm{I}} \\
\text { (per cent) }\end{array}$ & $\begin{array}{c}m_{\mathrm{O}} \\
\text { (per cent) }\end{array}$ & $Z_{\star}[1]$ & $\chi^{2}$ & $\begin{array}{l}\text { adev } \\
\text { (mag) }\end{array}$ & $A_{v}$ \\
\hline \multicolumn{11}{|c|}{ HRG 2304 (NED02) } \\
\hline-0.73 & 2.2 & 20.8 & 77.3 & 0.0 & 2.9 & 97.1 & 0.036 & 1.0 & 3.25 & 0.21 \\
\hline-0.49 & 0.0 & 12.1 & 87.3 & 0.0 & 1.0 & 99.0 & 0.026 & 1.0 & 3.13 & 0.42 \\
\hline-0.24 & 0.0 & 10.2 & 89.2 & 0.0 & 0.8 & 99.2 & 0.027 & 1.1 & 3.36 & 0.69 \\
\hline-0.08 & 0.0 & 10.6 & 89.4 & 0.0 & 0.8 & 99.2 & 0.027 & 1.1 & 3.24 & 0.93 \\
\hline 0.00 & 0.0 & 7.4 & 91.8 & 0.0 & 0.6 & 99.4 & 0.029 & 1.2 & 3.78 & 1.12 \\
\hline 0.16 & 0.0 & 9.5 & 90.3 & 0.0 & 0.7 & 99.3 & 0.027 & 1.3 & 3.43 & 1.22 \\
\hline 0.41 & 0.0 & 10.1 & 90.3 & 0.0 & 0.8 & 99.2 & 0.028 & 1.0 & 3.49 & 1.46 \\
\hline 0.65 & 4.9 & 2.5 & 92.2 & 0.0 & 0.2 & 99.8 & 0.028 & 1.0 & 3.68 & 1.69 \\
\hline 0.98 & 9.3 & 0.0 & 90.6 & 0.0 & 0.0 & 100.0 & 0.027 & 0.8 & 3.77 & 1.94 \\
\hline 1.22 & 6.4 & 0.0 & 94.1 & 0.1 & 0.0 & 99.9 & 0.025 & 0.9 & 3.86 & 1.95 \\
\hline 1.47 & 9.1 & 0.0 & 88.7 & 0.1 & 0.0 & 99.9 & 0.026 & 1.1 & 4.50 & 1.97 \\
\hline 1.80 & 11.2 & 0.0 & 86.5 & 0.1 & 0.0 & 99.9 & 0.028 & 0.8 & 5.09 & 1.88 \\
\hline \multicolumn{11}{|c|}{ AM 1646-795 (NED01) } \\
\hline-2.13 & 7.4 & 13.5 & 80.6 & 0.1 & 1.9 & 98.0 & 0.031 & 0.7 & 3.76 & $\overline{0.00}$ \\
\hline-1.72 & 4.9 & 21.4 & 73.8 & 0.0 & 3.2 & 96.7 & 0.032 & 0.8 & 3.57 & 0.00 \\
\hline-1.22 & 1.6 & 6.6 & 89.9 & 0.0 & 0.5 & 99.5 & 0.025 & 1.1 & 3.47 & 0.00 \\
\hline-0.74 & 0.0 & 7.6 & 89.8 & 0.0 & 0.6 & 99.4 & 0.028 & 1.6 & 3.31 & 0.32 \\
\hline-0.49 & 0.0 & 7.3 & 91.6 & 0.0 & 0.6 & 99.4 & 0.029 & 1.3 & 3.35 & 0.55 \\
\hline-0.16 & 1.7 & 3.1 & 92.4 & 0.0 & 0.2 & 99.8 & 0.030 & 1.5 & 3.31 & 0.90 \\
\hline 0.00 & 1.8 & 4.5 & 92.4 & 0.0 & 0.3 & 99.7 & 0.032 & 1.4 & 3.42 & 1.06 \\
\hline 0.24 & 3.8 & 1.6 & 92.1 & 0.0 & 0.1 & 99.9 & 0.032 & 1.5 & 3.30 & 1.30 \\
\hline 0.65 & 1.7 & 3.0 & 94.3 & 0.0 & 0.4 & 99.6 & 0.019 & 1.5 & 4.45 & 1.84 \\
\hline 2.53 & 13.6 & 0.0 & 78.1 & 0.2 & 0.0 & 99.8 & 0.020 & 0.6 & 6.51 & 2.20 \\
\hline
\end{tabular}

Notes. [1] Abundance by mass with $Z_{\odot}=0.02$.

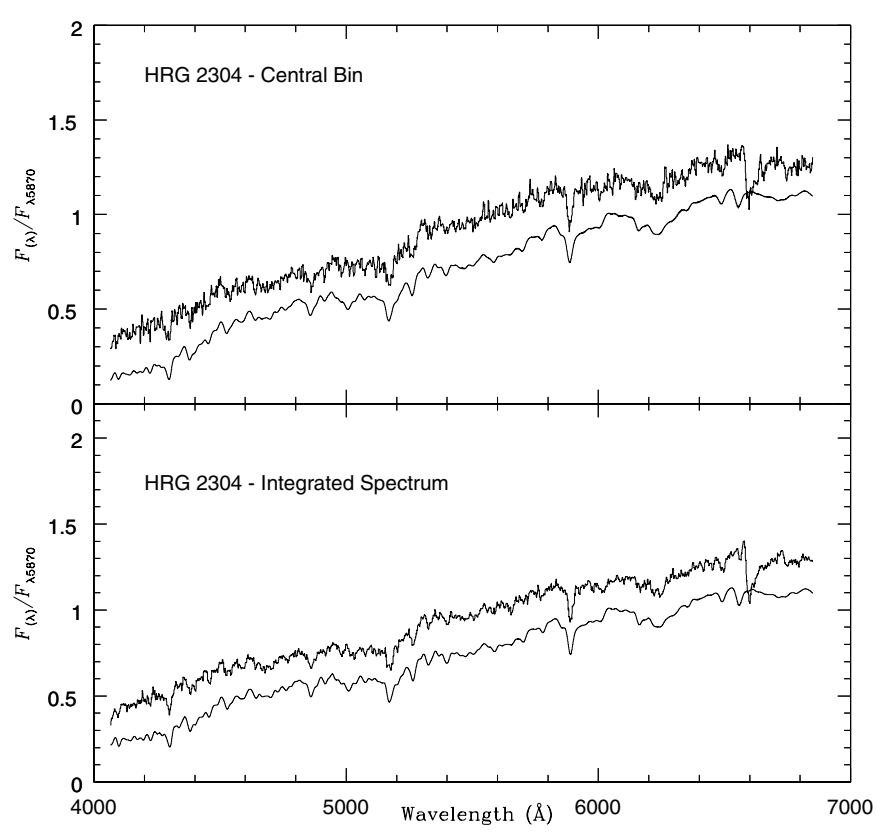

Fig. 7. Stellar population synthesis for HRG 2304. Top panel: central bin spectrum corrected for reddening and the synthesized spectrum (shifted down by a constant). Bottom panel: same as for the top panel but for the integrated spectrum of the galaxy.

components from $-22^{\prime \prime} 0$ to $22^{\prime \prime} 0$ around the kinematic center are receding from us.

The stellar formation history of both galaxies were well extracted by the stellar population synthesis code STARLIGHT (see Figs. 7 and 8). The synthesis results in flux fraction as a function of the distance to the center of each galaxy show that the spatial variation in the contribution of the stellar-population

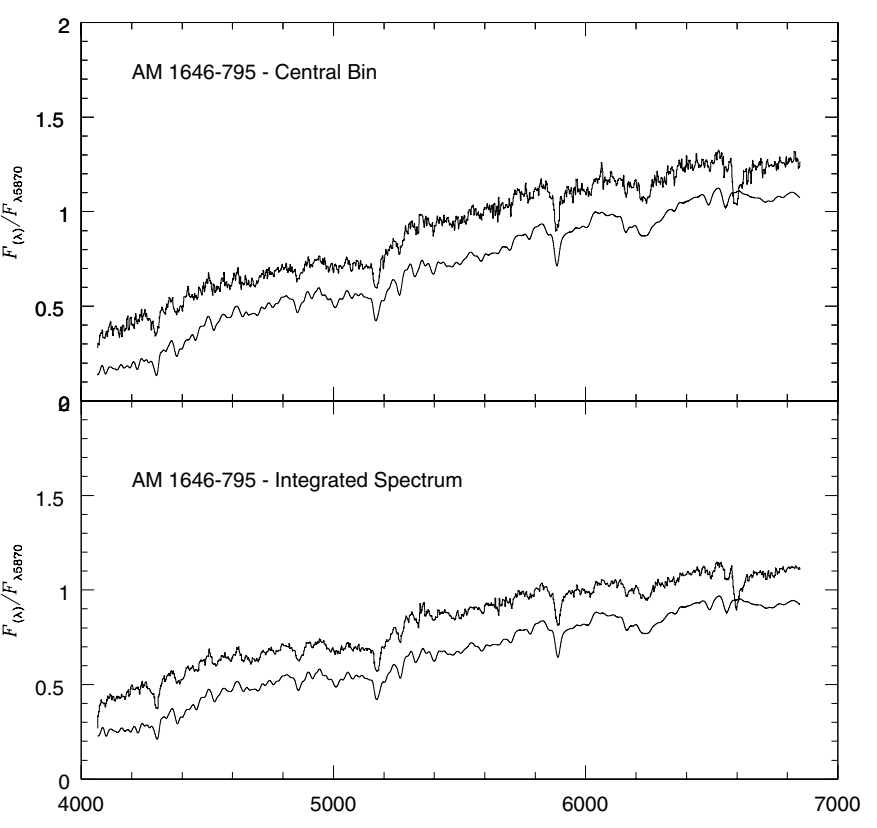

Fig. 8. Stellar population synthesis for AM 1646-795 (NED01). Top panel: central bin spectrum corrected for reddening and the synthesized spectrum (shifted down by a constant). Bottom panel: same as for the top panel but for the integrated spectrum of the galaxy.

components in both objects are dominated by an old stellar population with ages between $2 \times 10^{9}<t \leq 13 \times 10^{9} \mathrm{yrs}$. The latter confirms that both galaxies are early-type objects (ellipticallike galaxies and/or poor-gas S0 galaxies with an elliptical companion). The stellar population synthesis indicated that there is a small, but non-negligible, fraction of young stars (see first panel of Figs. 9 and 10). Those young stars are principally 


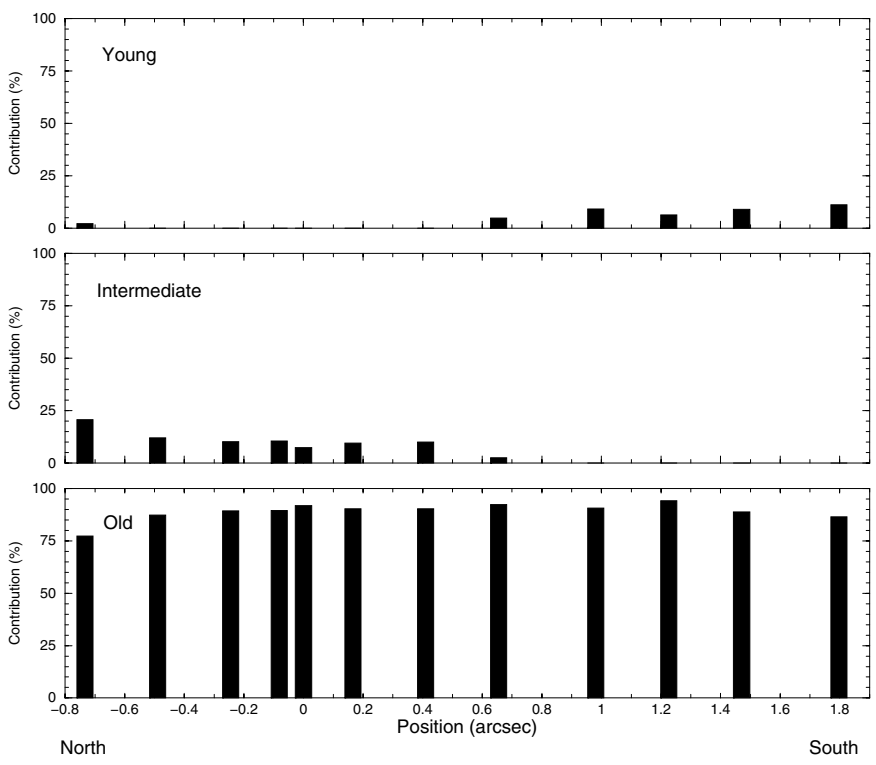

Fig. 9. Synthesis results in flux fractions as a function of the distance to the center of HRG 2304.

in HRG 2304 in the direction of the "contact" region between both galaxies, the S-region in HRG 2304 and the N-region in AM 1646-795 (NED01). Studies based on absoption line spectroscopy and studies of the fundamental plane favor a "frosting" model in which early-type galaxies consist of an old base population with a small amount of younger stars (Trager et al. 2000; Gebhart et al. 2003; and Schiavon 2007). Besides, data on earlytype galaxies which exhibit strong ultraviolet excess, polycyclic aromatic hydrocarbon emission and infrared excess, are interpreted as a possible result of recent low-level star formation (Yi et al. 2005; Rich et al. 2005; Schawinski et al. 2007; Kaviraj et al. 2007; Temi et al. 2009; Young et al. 2009; and Salim \& Rich 2010). Studies based in spatially resolved spectroscopy by Shapiro et al. (2010) and Kuntschner et al. (2010) find that star formation in early-type galaxies happens exclusively in fastrotating objects and occurs in two different contexts: (a) objects with widespread young stellar population associated with a high molecular gas content; (b) objects with disk and/or ring morphology. The latter could be explained by rejuvenation in previously quiescent stellar systems (Shapiro et al. 2010; Kuntschner et al. 2010), and seems to be suitable to explain HRG 2304.

In spite of the lack of results between 1-2 arcsec in the lower panel of Fig. 10, the old stellar population distribution appears homogeneous for both galaxies (see also Fig. 9). The intermediate population appears to be asymmetrically distributed in both galaxies (see the middle panel of Figs. 9 and 10). HRG 2304 seems to be different from other morphologically-similar objects which show nuclear activity and recent burst of star formation in the ring. As an example, the apparently morphologically-related ring galaxy NGC 985 shows Seyfert 1 activity, and the observations support the hypothesis that it contains two closely spaced nuclei as a result of the collision of two galaxies: a disk galaxy with an elliptical/spheroidal one, both or one of them with significant molecular and dust content. Different from HRG 2304, the ring galaxy NGC 985 is an object which has been extensively studied because of its peculiar characteristics (see NED; Appleton \& Struck-Marcell 1996; Arribas et al. 1999; Appleton et al. 2002; Krongold et al. 2005, among others. On the other hand, HRG 2304 seems to be more closely related to the ring galaxy FM 47-02, an early-type system which seems to originate

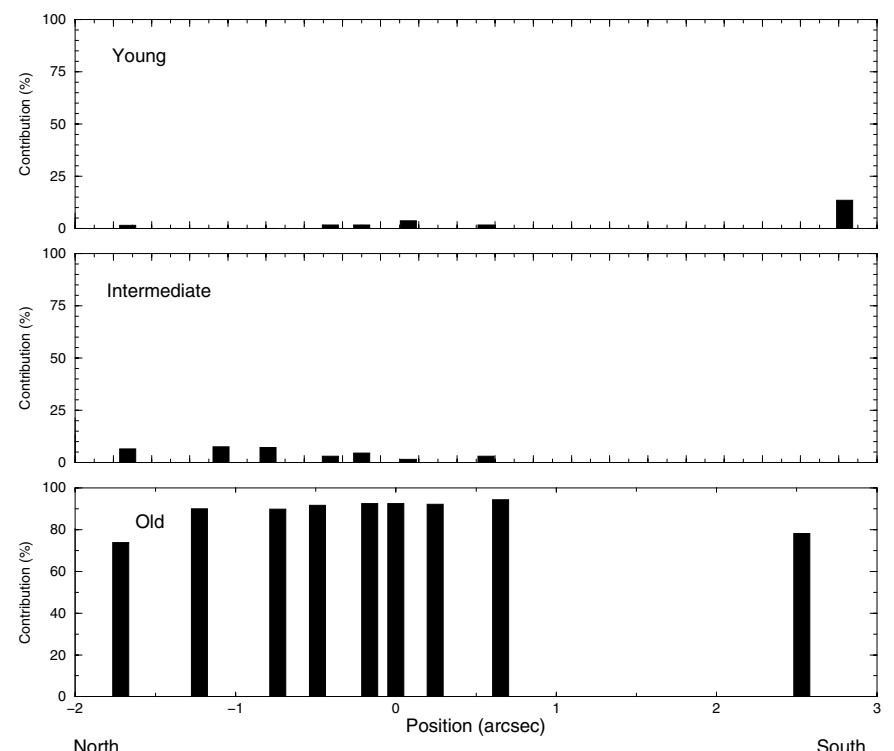

Fig. 10. Synthesis results in flux fractions as a function of the distance to the center of AM 1646-795 (NED01).

in the tidal interaction of a probable E/S0 galaxy with an elliptical E4 companion (Faúndez-Abans et al. 2010, 2011, in prep.).

The ring-like structure of HRG 2304 was probably triggered by a wave of enhanced density moving outwards, resulting only in the redistribution of the old stellar population of this galaxy. This structure resembles the asymmetric distribution of the galaxy contents seen in the simulations of slightly off-center collisions of Appleton \& Struck-Marcell (1987).

The roughly-derived ellipticity of the ring-like structure of HRG 2304 is 0.16 with an adopted radius of $7.5 \mathrm{kpc}$. There are some structures inside and around the ring, with a few of them marked by "a" in the lower panel of Fig. 3 (those are smooth clumps). We have also highlighted: the bright arc section of the ring "b"; the distorted bulge region of AM 1646-795 (NED01) "c", in the direction of HRG 2304; the fanlight structure "e", associated with AM 1646-795 (NED01); and a bright satellite candidate "d" (a more compact structure). In the first panel of Fig. 3, a section of the ring-like structure encompassing a few of its principal components is drawn. The related structures together with the ring around HRG 2304 are evidence of tidal interaction of both objects. The morphological and late-type star spectral characteristics, dominated by old stellar population in both objects and the off-center nucleus of HRG 2304 in the direction of NED01 (off-sets: 1'.92 $\mathrm{N} \rightarrow \mathrm{S}$ and 0.'48 $\mathrm{W} \rightarrow \mathrm{E}$ direction), suggest that HRG 2304 can be proposed as candidate of Solitaire ring galaxy in its early stages of interaction with the companion.

\section{Conclusions}

In this work, we report observations of the peculiar Ring Galaxy HRG 2304, which was previously classified as an elliptical-type ring galaxy by FAOA. Our work is based on low-resolution spectroscopic observations in the optical band to highlight some spectral characteristics of this object and its companion AM 1646-795 (NED01).

In summary, the results of our work on HRG 2304 are:

- The spectra of both galaxies show features that are characteristic of late-type stars. No star-forming regions and no nuclear ionization sources were detected. 
- The calculated heliocentric systemic velocity for HRG 2304 is $12449 \pm 40 \mathrm{~km} \mathrm{~s}^{-1}(z=0.0415)$, and for AM 1646-795 (NED01) it is $12430 \pm 40 \mathrm{~km} \mathrm{~s}^{-1}(z=0.0415)$; the latter is in agreement with early values in the literature.

- The calculated masses are $0.71 \times 10^{11} M_{\odot}$ for HRG 2304 and $1.50 \times 10^{11} M_{\odot}$ for AM 1646-795 (NED01), meaning that HRG 2304 has approximately half the mass of AM 1646-795 (NED01). Both objects are in a close encounter, and the separation between both galaxy centers is $13^{\prime \prime} .02$. The tidal interaction triggered the formation of a ring-like structure in HRG 2304 with a radius of approximately 8.'7 long.

- Both galaxies are at the same distance on the sky plane, and HRG 2304 seems to be face-on.

- In both galaxies, the spatial variation in the contribution of the stellar-population components are dominated by an old stellar population of $2 \times 10^{9}<t \leq 13 \times 10^{9} \mathrm{yr}$.

- There is a little, but non-negligible, fraction of young stars which is evident after the extraction by the stellar population synthesis code STARLIGHT. This suggests a probable rejuvenation in previously quiescent stellar systems remaining in both galaxies.

- The ring-like structure of HRG 2304 was probably triggered by the tidal wave moving outwards, resulting only in the redistribution of the old stellar population of this galaxy. The latter suggests that HRG 2304 did not have enough gas and dust to trigger star formation in regions compressed by the density wave.

Finally, the goal of this paper is to bring the first input in the study of the Solitaire-class of Ring Galaxies. The spectroscopic results and the morphological peculiarities of HRG 2304 and its companion AM 1646-795 (NED01), can be adequately interpreted as an ongoing stage of interaction. Both galaxies are early-type, the companion is elliptical, and the smooth distribution of the material around HRG 2304 and its off-center nucleus in the direction of AM1646-795 (NED01) characterize HRG 2304 as a candidate to Solitaire-type Ring Galaxy in its early-stage of ring formation. Despite the low resolution of the direct images and the uncertainties related to the possible group companionship and dynamics, we intend to conduct an exploratory study by employing numerical $\mathrm{N}$-body/hydrodynamical simulation to reconstruct this history and to predict the evolution of the tidal interaction. This exploration of collision parameters will certainly contribute to a better understanding of the Solitaire forming process, probably in the sense of constraining the group of physical parameters and boundary conditions.

Acknowledgements. This work was partially supported by the Brazilian Ministério da Ciência e Tecnologia (MCT), Laboratório Nacional de Astrofísica (MCT/LNA), and Universidade do Vale do Paraíba - UNIVAP. A. C. Krabbe thanks the support of FAPESP, process 2010/01490-3. We also thank Ms. Alene Alder-Rangel for editing the English in this manuscript. This research made use of the NASA/IPAC Infrared Science Archive, which is operated by the Jet Propulsion Laboratory, California Institute of Technology, under contract with the National Aeronautics and Space Administration.

\section{References}

Appleton, P. N., \& Struck-Marcell, C. 1987, ApJ, 318, 103

Appleton, P. N., \& Struck-Marcell, C. 1996, in Fundamentals of Cosmic Physics $16 / 2,111$

Appleton, P. N., Charmandaris, V., Gao, Y., Combes, F., et al. 2002, ApJ, 566, 682

Arp, H. C., \& Madore, B. F. 1977, QJl.R. Astron. Soc., 18, 234

Arp, H. C., \& Madore, B. F. 1986, Catalogue of Southern Peculiar galaxies and Associations, Vol. I, Positions and Descriptions (Toronto: Clarke-Irwin)

Arribas, S., Mediavilla, E., del Burgo, C., \& García-Lorenzo, B. 1999, ApJ, 511, 680

Asari, N. V., Cid Fernandes, R., Stasińska, G., et al. 2007, MNRAS, 381, 263

Baldwin, J. A., \& Stone, R. P. S. 1984, MNRAS, 206, 241

Bruzual, G., \& Charlot, S. 2003, MNRAS, 344, 1000

Cardelli, J. A., Clayton, G. C., \& Mathis, J. S. 1989, ApJ, 345, 245

Chabrier, G. 2003, PASP, 115, 763

Cid Fernandes, R., Gu, Q., Melnick, J., et al. 2004, MNRAS, 355, 273

Cid Fernandes, R., Mateus, A., Sodré, L., Stasińska, G., \& Gomes, J. M. 2005, MNRAS, 358, 363

Dennefeld, M., \& Materne, J. 1980, The ESO Messenger, 21, 29

Faúndez-Abans, M., \& de Oliveira-Abans, M. 1998a, A\&AS, 129, 357

Faúndez-Abans, M., \& de Oliveira-Abans, M. 1998b, A\&AS, 128, 289

Faúndez-Abans, M., Cuevas, H. L., \& Hertling, G. P. 1994, A\&AS, 104, 1

Faúndez-Abans, M., de Oliveira-Abans, M., Fernandes, I. F., et al. 2010, Proceedings of the Workshop OPD, SOAR and Gemini, Campos do Jordão, Brazil

Gebhardt, K., Faber, S. M., Koo, D. C., et al. 2003, ApJ, 597, 239

Gordon, K. D., Clayton, G. C., Misselt, K. A., Landolt, A. U., \& Wolff, M. J. 2003, ApJ, 594, 279

Hamuy, M., Walker, A. R., Suntzeff, N. B., et al. 1992, PASP, 104, 533

Hamuy, M., Suntzeff, N. B., Heathcote, S. R., et al. 1994, PASP, 106, 566

Kaviraj, S., Schawinski, K., Devriendt, J. E. G., et al. 2007, ApJS, 173, 619

Krabbe, A. C., Pastoriza, M. G., Winge, C., et al. 2011, MNRAS, submitted

Krongold, Y., Nicastro, F., Elvis, M., et al. 2005, ApJ, 620, 165

Kuntschner, H., Emsellem, E., Bacon, R., et al. 2010, MNRAS, 408, 97

Landolt, A. U. 1992, AJ, 104, 340

Lynds, R., \& Toomre, A. 1976, ApJ, 209, 382

Mateus, A., Sodré, L., Cid Fernandes, R., et al. 2006, MNRAS, 370, 721

Mitronova, S. N., Karachentsev, I. D., Karachentseva, V. E., et al. 2004, Bulletin of the Special Astrophysical Observatory, 57, 5

Paturel, G., Vauglin, I., Petit, C., et al. 2005, A\&A, 430, 751

Rich, R. M., Salim, S., Brinchmann, J., et al. 2005, ApJ, 619, L107

Salim, S., \& Rich, R. M. 2010, ApJ, 714, L290

Shapiro, K. L., Falcon-Barroso, J., van de Ven, G., et al. 2010, MNRAS, 402, 2140

Schawinski, K., Kaviraj, S., Khochfar, S., et al. 2007, ApJS, 173, 512

Schiavon, R. P. 2007, ApJS, 171, 146

Temi, P., Brighenti, F., \& Mathews, W. G. 2009, ApJ, 695, 1

Theys, J. C., \& Spiegel, E. A. 1976, ApJ, 208, 650

Theys, J. C., \& Spiegel, E. A. 1977, ApJ, 212, 616

Tranger, S. C., Faber, S. M., Worthey, G., \& González, J. J. 2000, AJ, 119, 1645

Yi, S. K., Yoon, S. J., Kaviraj, S., et al. 2005, ApJ, 619, L111

Young, L. M., Bendo, G. J., \& Lucero, D. M. 2009, AJ, 137, 3053 